\title{
Effect of noise on the power spectrum of passively mode-locked lasers
}

\author{
Danny Eliyahu, Randal A. Salvatore, and Amnon Yariv \\ California Institute of Technology, M/S 128-95, Pasadena, California 91125
}

Received October 26, 1995; revised manuscript received May 21, 1996

\begin{abstract}
We analyze the effects of noise on the power spectrum of pulse trains generated by a continuously operating passively mode-locked laser. The shape of the different harmonics of the power spectrum is calculated in the presence of correlated timing fluctuations between neighboring pulses and in the presence of amplitude fluctuations. The spectra at the different harmonics are influenced mainly by the nonstationary timing-jitter fluctuations; amplitude fluctuations slightly modify the spectral tails. Estimation of the coupling term between the longitudinal cavity modes or the effective saturable absorber coefficient is made from the timingjitter correlation time. Experimental results from an external cavity two-section semiconductor laser are given. The results show timing-jitter fluctuations having a relaxation time much longer than the repetition period. (C) 1997 Optical Society of America. [S0740-3224(97)02801-4]
\end{abstract}

\section{INTRODUCTION}

Mode locking is a common technique employed to produce ultrashort optical pulses from lasers over a wide range of wavelengths. Such lasers serve as reliable sources of picosecond and subpicosecond pulses. Different schemes can produce locking of the phases of the longitudinal cavity modes. However, there is a fundamental difference between the characterization of noise in passively modelocked lasers and that in externally-locked mode-locked lasers. Actively (or hybrid) mode-locked lasers, for example, are driven by a stable external frequency reference, usually having much less jitter than the laser. In this case, the timing fluctuations are related to the modulator signal and can be treated as a stationary process. A theoretical description for the low frequencies of the power spectrum under the assumption of stationary processes, given by von der Linde, ${ }^{1}$ is typically used to measure the timing-jitter and intensity rms fluctuations. This theory revealed spectral characteristics that are commonly referred to as a spike and a pair of pedestals, with the spike resulting from a delta-function-like shape at each harmonic owing to the external locking source. One pedestal, or side band, at each harmonic is due to amplitude fluctuations and the second is due to timing jitter fluctuations. The latter is harmonic-number dependent. In a separate publication the theory for actively mode-locked lasers was extended ${ }^{2}$ and the entire power spectrum in the presence of correlated amplitude, pulse shape, and timing-jitter fluctuations was analyzed. For more than a decade attempts were made to apply this model to the fundamentally different case of measuring the noise of nonstationary timing-jitter fluctuations as in the case of passively mode-locked lasers; however, the model will not adequately explain the passively modelocked laser results.

In contrast to active mode locking, a passively modelocked laser is a free-running laser, where the modulation is produced internally. Therefore timing fluctuations are cumulative, with the position of the $n$th pulse dependent on the position of the $(n-1)$ th pulse, and are described as a nonstationary processes. ${ }^{3}$ In some applications such as optical data transmission, in which the absolute phase of the repetition cycle may be important, these fluctuations of the phase may make passive mode-locking undesirable. However, in other cases, such as time-resolved spectroscopy, pump-probe measurements, electro-optic sampling, or free space and fiber ranging, in which only the relative pulse timing fluctuations may be important, it is crucial that the fluctuations in the mode-locked laser's period be studied more thoroughly.

The nonstationary statistics of the timing jitter gives a direct-detection spectrum without the characteristic spikes. Not only is the spike absent at each harmonic, but a completely different relationship of the relative peaks of the harmonics and their relative shapes and widths are expected. Therefore, for a proper understanding of the spectral characteristics and a useful characterization of passive mode-locking for those applications and others, consideration of the noise statistics is essential. Our purpose in this paper is to calculate the form of the power spectrum of pulse trains generated by passively mode-locked lasers. The theory is based on the basic characteristic of timing-jitter noise, i.e., on phenomenological characterization of the processes in passive mode locking, for which a basic physical approach is used. Therefore this theory is not restricted to a specific structure or technique of passive mode locking and offers physical insight into the statistical processes in passively mode-locked lasers. Experimental results from an external cavity two-section semiconductor laser, supporting the theory, are presented. The results show timing-jitter fluctuations having a relaxation time much longer than the repetition period.

\section{MODEL}

We employ the technique of radio-frequency spectrum analysis to investigate random fluctuations in trains of 
ultra-short pulses. As typically implemented, this technique is based on recording the power spectrum of the laser intensity by use of a fast photodiode and an electronic spectrum analyzer.

We start with the assumption that the detected intensity $I_{\Delta t}$ of a train of $2 N+1$ pulses can be described in the time domain as

$$
I_{\Delta t}(t)=\sum_{n=-N}^{N} f_{n}\left(t-T_{n}\right),
$$

where $f_{n}$ is the $n$th pulse intensity envelope in the train, which occurs at time $T_{n}$. The time duration of the $2 N$ +1 pulse train is $\Delta t$. Equation (1) assumes nonoverlapping electric field envelopes of neighboring pulses in the train.

The power spectrum of the detected photo current $P_{I}(\omega)$ is given in terms of $I_{\Delta t}(\omega),{ }^{2,4}$

$$
P_{I}(\omega)=\lim _{\Delta t \rightarrow \infty} \frac{1}{\Delta t}\left\langle I_{\Delta t}(\omega) I_{\Delta t}^{*}(\omega)\right\rangle
$$

where

$$
I_{\Delta t}(\omega)=\int_{-\Delta t / 2}^{\Delta t / 2} I_{\Delta t}(t) e^{i \omega t} \mathrm{~d} t .
$$

$I_{\Delta t}^{*}(\omega)$ is the complex conjugate of $I_{\Delta t}(\omega)$ and $\langle\ldots\rangle$ denotes an ensemble average.

Without any loss of generality, we can assume that the individual event $f_{n}(t)$ occurs over a short time compared with the observation period $\Delta t$, so the integration limits can be taken as $-\infty$ to $\infty$ instead of $-\Delta t / 2$ to $\Delta t / 2$. Substituting Eq. (1) into Eq. (3) gives

$$
I_{\Delta t}(\omega)=\sum_{n=-N}^{N} F_{n}(\omega) \exp \left(i \omega T_{n}\right),
$$

where

$$
F_{n}(\omega)=\int_{-\infty}^{\infty} f_{n}(t) \exp (i \omega t) \mathrm{d} t .
$$

Therefore the power spectrum of the pulse train is given by

$$
\begin{aligned}
P_{I}(\omega)= & \lim _{N \rightarrow \infty} \frac{1}{2 N+1} \sum_{n, m=-N}^{N}\left\langle F_{n}(\omega) F_{m}^{*}(\omega)\right. \\
& \left.\times \exp \left[i \omega\left(T_{n}-T_{m}\right)\right]\right\rangle .
\end{aligned}
$$

$F_{n}(\omega)$ describes the Fourier transform of the amplitude and the pulse shape of the intensity of the $n$th pulse in the train, and the timing-jitter fluctuation manifests itself in the exponent.

\section{TIMING-JITTER FLUCTUATIONS}

Random variations of the time interval between successive pulses in the train are given by

$$
T_{n}=n T+\delta T_{n}
$$

where $T$ represents the average pulse repetition period and $\delta T_{n}$ is a random variable describing the fluctuations relative to the time $n T$ of the $n$th pulse in the train. Therefore

$$
\begin{aligned}
P_{I}(\omega)= & \lim _{N \rightarrow \infty} \frac{1}{2 N+1} \sum_{n, m=-N}^{N} \exp [i \omega T(n-m)] \\
& \times\left\langle F_{n}(\omega) F_{m}^{*}(\omega) \exp \left[i \omega\left(\delta T_{n}-\delta T_{m}\right)\right]\right\rangle .
\end{aligned}
$$

Different types of processes govern the timing-jitter fluctuations for different mode-locked systems. In actively mode-locked lasers, because of the external stable restoring force of the active modulation, the timing fluctuations are described by stationary processes

$$
\left\langle\left(\delta T_{n}-\delta T_{m}\right)^{2}\right\rangle=2\left[G_{T}(0)-G_{T}(|n-m|)\right],
$$

where $\delta T_{n}$ is related to the modulator signal and $G_{T}(k)$ $=\left\langle\delta T_{0} \delta T_{k}\right\rangle$ is the autocorrelation of the timing-jitter fluctuations. The timing fluctuations as given in Eq. (9) are finite for any $n, m$ and bounded by $2 G_{T}(0)$ since $\left|G_{T}(k)\right| \leqslant G_{T}(0)$. In Fig. 1(a), a plot of a typical form of $\left\langle\left(\delta T_{n}-\delta T_{m}\right)^{2}\right\rangle$ for stationary timing jitter noise is shown. For short time scales compared with the correlation time $\tau_{t}$ [the decay time of $\left.G_{T}(|n-m|)\right]$, i.e., $\mid n$ $-m \mid T \ll \tau_{t}$, the average is small, but at long time scales this value is constant and approaches the value $2 G_{T}(0)$. This characterization of $\left\langle\left(\delta T_{n}-\delta T_{m}\right)^{2}\right\rangle$ is the key to the existence of the spikes in the spectra of different harmonics also in the presence of noise. Therefore the timingjitter fluctuations manifest themselves as side bands or pedestals around the spikes at multiples of the fundamental repetition frequency in the power spectrum. These side bands differ as a function of harmonic number. For low frequencies in the power spectrum they are proportional to the square of the harmonic number. Their power comes at the expense of the discrete Dirac $\delta$ functions, corresponding to the noiseless laser. Because of both noise and finite detector bandwidth, the discrete lines are attenuated and roll off at high frequencies. Their appearance is a direct result of the stationary processes of timing fluctuations. The amplitude noise is reflected by harmonic-number-independent side bands., ${ }^{1,2}$

In passive mode locking an external timing source is not required for the laser's operation. Instead, the modulation is produced internally and self consistently through the action of the optical pulse on the gain and the absorber media because of its own internal nonlinearities. In this case the timing-jitter fluctuations do not obey a stationary process. In the absence of an external modulator, the timing of each pulse depends on that of the previous one and its fluctuations result from the sum of many processes. For nonstationary timing jitter and for small intensity and pulse shape fluctuations, the power spectrum is affected mainly by the timing noise. Therefore with Eq. (8) the power spectrum may be written as

$$
\begin{aligned}
P_{I}(\omega)=\mid & |F(\omega)|^{2} \lim _{N \rightarrow \infty} \frac{1}{2 N+1} \sum_{n, m=-N}^{N} \exp [i \omega T(n-m)] \\
& \times\left\langle\exp \left[i \omega\left(\delta T_{n}-\delta T_{m}\right)\right]\right\rangle
\end{aligned}
$$



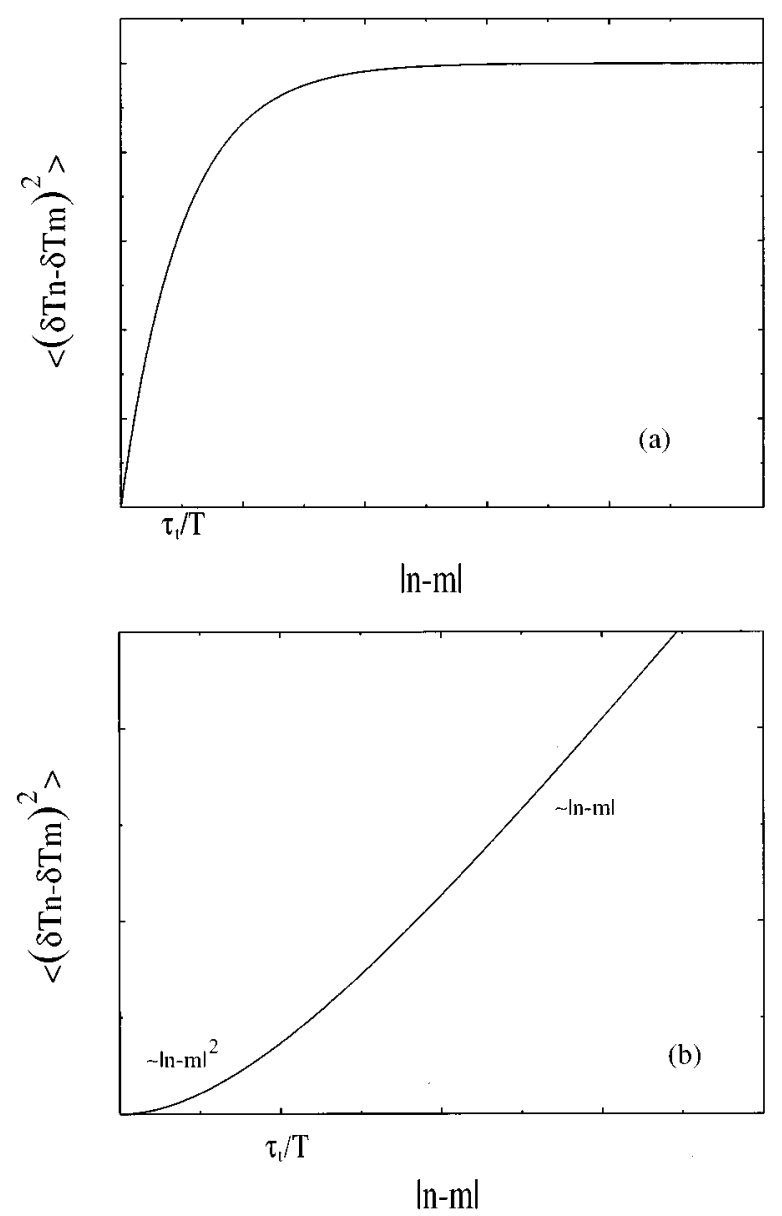

Fig. 1. Timing-jitter noise in the two opposed cases. (a) The stationary fluctuations in actively mode-locked lasers. For long time scale compared with the fluctuations, correlation time $\tau_{t}$ has a bounded $\left\langle\left(\delta T_{n}-\delta T_{m}\right)^{2}\right\rangle$ that reaches the constant value $2 G_{T}(0)$. (b) The nonstationary fluctuations in passively modelocked lasers. For short time compared with the correlation time, $\tau_{t}$ has $\left\langle\left(\delta T_{n}-\delta T_{m}\right)^{2}\right\rangle$ that shows a time-squared $(\sim \mid n$ $\left.-\left.m\right|^{2}\right)$ dependence while for long time $\left(\gg \tau_{t}\right)$ it is unbounded and reveals a linear dependence on time $(\sim|n-m|)$.

where $F(\omega)=F_{n}(\omega)$ drops only gently at low frequencies of the power spectrum,

$$
\delta T_{n}-\delta T_{m}=\operatorname{sgn}(n-m) \sum_{i=\min (n, m)+1}^{\max (n, m)} \Delta T_{i} .
$$

$\Delta T_{i}=\delta T_{i}-\delta T_{i-1}$ describes the timing-jitter fluctuation between successive neighboring pulses, and $\operatorname{sgn}(x)$ is the sign of $x$. The effects of strong amplitude fluctuations on the power spectrum are discussed in Section 5 .

In contrast to the case employing a stable external modulator, the timing fluctuations $\delta T_{n}-\delta T_{m}$ are unbounded as a function of $|n-m|$. Assuming Gaussian probability distribution functions for the timing jitter, the power spectrum is given by

$$
\begin{aligned}
P_{I}(\omega)= & |F(\omega)|^{2} \lim _{N \rightarrow \infty} \frac{1}{2 N+1} \sum_{n, m=-N}^{N} \exp [i \omega T(n-m)] \\
& \times \exp \left[-\frac{\omega^{2}}{2}\left\langle\left(\delta T_{n}-\delta T_{m}\right)^{2}\right\rangle\right]
\end{aligned}
$$

where

$$
\left\langle\left(\delta T_{n}-\delta T_{m}\right)^{2}\right\rangle=\sum_{i, j=\min (n, m)+1}^{\max (n, m)}\left\langle\Delta T_{i} \Delta T_{j}\right\rangle .
$$

In contrast to $\delta T_{n}, \Delta T_{i}$ does not depend on the time origin. Translation in time does not change the statistical characteristics of $\Delta T_{i}$, which is stationary. We assume that the timing fluctuation between neighboring pulses $\Delta T_{i}$ are characterized by some correlation time $\tau_{t}$. For simplicity, a negative exponential decay of these correlations is assumed. Therefore we may write

$$
\left\langle\Delta T_{i} \Delta T_{j}\right\rangle=\left\langle\Delta T^{2}\right\rangle \exp \left[-|i-j| T / \tau_{t}\right],
$$

where $\left\langle\Delta T^{2}\right\rangle=\left\langle\Delta T_{i}^{2}\right\rangle$ is assumed to be time independent. When we substitute Eq. (14) into Eq. (13), the sum over the indices $i, j$ gives

$$
\begin{aligned}
\left\langle\left(\delta T_{n}-\delta T_{m}\right)^{2}\right\rangle= & 2\left\langle\Delta T^{2}\right\rangle \frac{\exp \left(-T / \tau_{t}\right)}{\left[1-\exp \left(-T / \tau_{t}\right)\right]^{2}} \\
& \times\left[|n-m| \frac{1-\exp \left(-2 T / \tau_{t}\right)}{2 \exp \left(-T / \tau_{t}\right)}-1\right. \\
& \left.+\exp \left(-|n-m| T / \tau_{t}\right)\right]
\end{aligned}
$$

Figure 1(b) shows a typical form of $\left\langle\left(\delta T_{n}-\delta T_{m}\right)^{2}\right\rangle$ for timing-jitter fluctuations in the nonstationary case. For short time scales compared with the correlation time $(\mid n$ $\left.-m \mid T \ll \tau_{t}\right)$ the dependence is on the time squared $\left(\sim|n-m|^{2}\right)$, whereas for long time scales, in contrast to stationary noise, the average is unbounded and reveals a linear dependence on time $(\sim|n-m|)$. The absence of spikes or delta functions in the spectra is a direct result of the unbounded characterization of $\left\langle\left(\delta T_{n}-\delta T_{m}\right)^{2}\right\rangle$ for long time scales between pulses. In what follows, we use these properties to give an analytical form for the spectra at different harmonics of the power spectrum.

One can find the shape of the power spectrum for different limiting cases. First we assume that the fluctuations are due to white noise, i.e., the correlation time is smaller than the repetition time $\left(\tau_{t} \ll T\right)$. In this case each pulse suffers nearly independent timing fluctuation

$$
\left\langle\Delta T_{i} \Delta T_{j}\right\rangle \simeq\left\langle\Delta T^{2}\right\rangle \delta_{i, j},
$$

where $\delta_{i, j}=1$ if $i=j$ and zero otherwise. Therefore assuming $\tau_{t} / T \ll 1$ in Eq. (15), or using Eqs. (13) and (16), gives

$$
\left\langle\left(\delta T_{n}-\delta T_{m}\right)^{2}\right\rangle=\left\langle\Delta T^{2}\right\rangle|n-m| .
$$

Substituting Eq. (17) into (12) gives the power spectrum form

$$
P_{I}(\omega)=|F(\omega)|^{2} \frac{\sinh \left(\omega^{2}\left\langle\Delta T^{2}\right\rangle / 2\right)}{\cosh \left(\omega^{2}\left\langle\Delta T^{2}\right\rangle / 2\right)-\cos (\omega T)} .
$$

For low frequency $\left(\omega^{2}\left\langle\Delta T^{2}\right\rangle \ll 1\right)$ the power spectrum is given approximately by the Lorentzian functions around multiples of the fundamental repetition frequency

$$
P_{I}(\omega)=|F(\omega)|^{2} \sum_{n=0}^{\infty} \frac{\omega^{2}\left\langle\Delta T^{2}\right\rangle}{\left(\omega^{2}\left\langle\Delta T^{2}\right\rangle / 2\right)^{2}+\left(\Delta \omega_{n} T\right)^{2}},
$$


where $\Delta \omega_{n}=\omega-2 \pi n / T$ is the frequency deviation (offset frequency) of the $n$th harmonic power spectrum. An important characteristic of this result is the Lorentzian shape with FWHM of $\Delta \omega_{W} \simeq \omega^{2}\left\langle\Delta T^{2}\right\rangle / \sqrt{2} T$, which is proportional to the square of the harmonic number. The peak values of the spectra of different harmonics decay as $1 / \omega^{2}$. The factor $|F(\omega)|^{2}$, which represents the pulse shape effects and the finite response of the detector system, forms an envelope for the peak value of the different harmonics. Usually this factor is unknown; therefore the items of greatest interest in the measurement are the FWHM of the spectra of different harmonics and their shapes.

The second case is of long correlation time $\left(\tau_{t} \gg T\right)$. In this case Eq. (15) has the form

$$
\begin{aligned}
\left\langle\left(\delta T_{n}-\delta T_{m}\right)^{2}\right\rangle= & 2\left\langle\Delta T^{2}\right\rangle\left(\frac{\tau_{t}}{T}\right)^{2}\left[|n-m| \frac{T}{\tau_{t}}-1\right. \\
& \left.+\exp \left(-|n-m| T / \tau_{t}\right)\right] .
\end{aligned}
$$

For time scales smaller than the correlation time $(\mid n$ - $m \mid T \ll \tau_{t}$ ) the fluctuations are highly correlated, and the pulse train is stable for this period of time with an almost constant repetition time $T+\Delta T$ (where $\left\langle\Delta T_{i} \Delta T_{j}\right\rangle$ $\simeq\left\langle\Delta T^{2}\right\rangle$ ). On the other hand, for time scales longer than the correlation time $\left(|n-m| T \gg \tau_{t}\right)$ the processes are diffusionlike, similar to the case of $T \gg \tau_{t}$ [see Eq. (17)]. Therefore the two limiting cases of Eq. (20) have the form

$$
\begin{aligned}
& \left\langle\left(\delta T_{n}-\delta T_{m}\right)^{2}\right\rangle \\
& =\left\langle\Delta T^{2}\right\rangle\left\{\begin{array}{cc}
|n-m|^{2} & |n-m| T \ll \tau_{t} \\
2|n-m| \frac{\tau_{t}}{T} & |n-m| T \gg \tau_{t}
\end{array} .\right.
\end{aligned}
$$

It is difficult to obtain an analytical expression for the power spectrum with Eq. (20); however, for frequencies of the order of $\omega^{2}\left\langle\Delta T^{2}\right\rangle\left(\tau_{t} / T\right)^{2} \sim 1$ or higher, the main contribution to the power spectrum is from the short time scale. Therefore, when we substitute the upper part of Eq. (21) into Eq. (12) and use the Poisson summation formula, the power spectrum for harmonic numbers higher than $n_{\min }=(\sqrt{2} / 2 \pi)\left(T / \tau_{t}\right)\left(T /\left\langle\Delta T^{2}\right\rangle^{1 / 2}\right)$ is given by

$$
P_{I}(\omega)=|F(\omega)|^{2}\left(\frac{2 \pi}{\omega^{2}\left\langle\Delta T^{2}\right\rangle}\right)^{1 / 2} \sum_{n=-\infty}^{\infty} \exp \left[-\frac{\left(\Delta \omega_{n} T\right)^{2}}{2 \omega^{2}\left\langle\Delta T^{2}\right\rangle}\right] .
$$

For small timing-jitter fluctuations $\left(\left\langle\Delta T^{2}\right\rangle \ll T\right)$, as is typical in passively mode-locked lasers, the main contribution to the $n$th harmonic number of the power spectrum is from the $n$th term in the summation of Eq. (22). Therefore spectra of the $n$th harmonic number of the power spectrum are given approximately by a Gaussian form with FWHM $\Delta \omega_{W} \simeq 2 \omega \sqrt{\left\langle\Delta T^{2}\right\rangle \ln 2} / T$, i.e., the width is proportional to the harmonic number. Note that the peak values of the different harmonics decay as $1 / \omega$ (in addition to the pulse shaping influence and other system dependence).

The spectra of lower frequencies, or harmonic numbers lower than $n_{\text {min }}$, are also influenced by long time scales

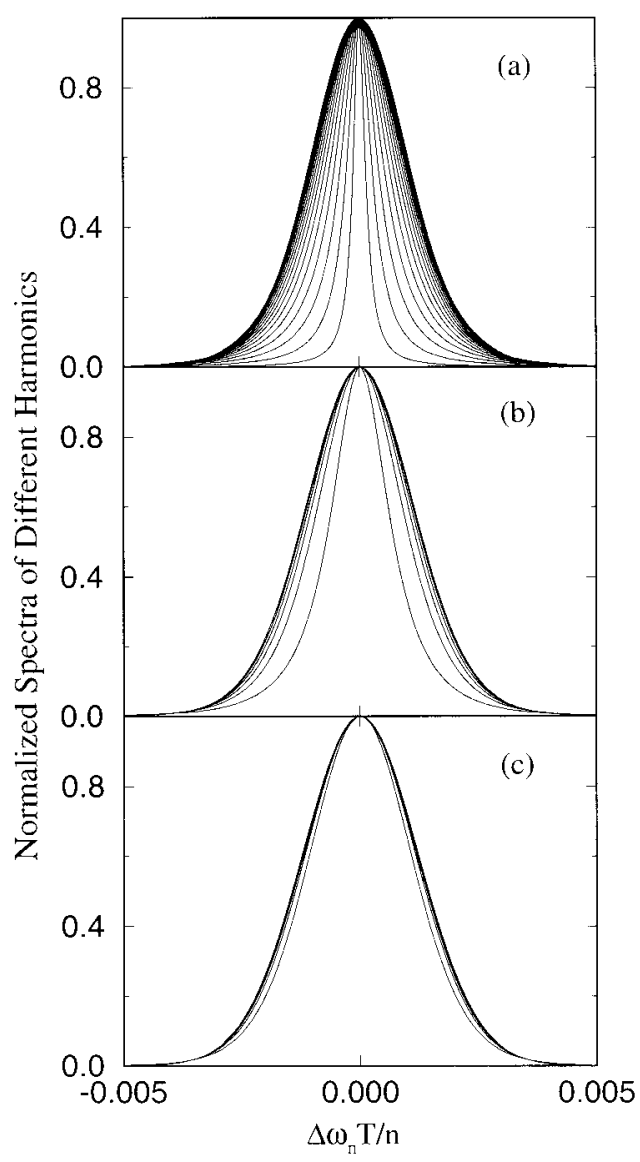

Fig. 2. Numerical calculation, based on Eqs. (10) and (15), of the normalized spectra around different harmonic numbers versus the scaled offset frequency for $\left\langle\Delta T^{2}\right\rangle^{1 / 2} / T=2 \times 10^{-4}$. (a) $\tau_{t} / T=100$, (b) $\tau_{t} / T=500$, (c) $\tau_{t} / T=2000$. The innermost curve in each part represents the spectra at the first harmonic. As the harmonic number increases, its spectrum becomes wider and approaches the Gaussian shape.

( $\left.|n-m| T>\tau_{t}\right)$, and the full expression of Eq. (20) should be considered in calculating the power spectrum. However, it is easy to verify that the influence of longer time scale fluctuations on lower frequencies of the power spectrum results in modification of the shapes toward Lorentzians and makes their FWHM's smaller. This effect leads to higher-order dependence of the FWHM spectra on the harmonic number. The power can vary between 1 and 2 depending on the power spectrum frequency, the noise strength $\left\langle\Delta T^{2}\right\rangle$, and the ratio $\tau_{t} / T$. Notice that $n_{\text {min }}$ depends on the timing-jitter rms fluctuation, the averaged repetition time, and the correlation time. For strong fluctuations and long correlation times, $n_{\text {min }}$ can even reach the value of 1 , which means that all the spectra of different harmonics of the power spectrum are described by a Gaussian shape with FWHM that grows linearly with the harmonic number.

Numerical calculations of the normalized spectra of different harmonics as a function of the scaled offset frequency $\Delta \omega_{n} T / n$ are presented in Fig. 2 (throughout this paper, normalization is employed such that the peak value is equal to unity). The calculation is obtained for a rms timing-jitter fluctuation of the order of $\left\langle\Delta T^{2}\right\rangle^{1 / 2} / T$ $=2 \times 10^{-4}$. Figure 2(a) describes the spectra of differ- 
ent harmonics for $\tau_{t} / T=100$. The innermost curve represents the spectrum of the first harmonic. As the harmonic number increases, its shape becomes wider and approaches the Gaussian shape as in Eq. (22). In this case $n_{\min } \simeq 11$; therefore harmonic numbers higher than 11 are scaled by the Gaussian shape.

Figures 2(b) and 2(c) describe the normalized spectra of different harmonics for $\tau_{t} / T=500$ and $\tau_{t} / T=2000$, respectively. As the correlation time increases, $n_{\text {min }}$ decreases and the spectra of a larger number of harmonics approach the Gaussian shape.

\section{CORRELATION TIME $\boldsymbol{\tau}_{t}$}

The correlation time $\tau_{t}$ results, most likely, from relaxation processes in the mode-locked laser, which are responsible for timing-jitter fluctuations. One of these processes (e.g., a random process, such as spontaneous emission or gain profile fluctuation) results in a change of the central optical frequency through the action of the gain on the optical spectra. Indeed, Haus and Mecozzi ${ }^{3}$ solved the time domain equation for the electric field envelope of mode-locked lasers operated with a fast saturable absorber. The calculation uses a soliton perturbation theory and shows that timing-jitter fluctuations result from changes in group velocity through changes in the group-velocity dispersion and its dependence on the angular carrier frequency. The timing-jitter fluctuations were found to have a behavior similar to that described in Eq. (20). The mean optical frequency was found to fluctuate as a damped process with relaxation time $\tau_{t}$ given by

$$
\frac{T}{\tau_{t}} \simeq \frac{g}{\Omega_{g}{ }^{2} \tau_{p}{ }^{2}},
$$

where $g$ is the saturated gain, $\Omega_{g}$ is its bandwidth, and $\tau_{p}$ is the pulse FWHM. Other components of timing-jitter noise consisting of white noise were found to be present, similar to Eq. (17). Equation (23) shows that, besides the dependence on gain properties, the relaxation time depends on the pulse width. This implies that more processes are involved in producing the relaxation time. That is, the relaxation time results from two or more processes. The gain of the pulse whose center frequency is off the gain-line center is nonuniform across the spectrum and pushes the spectrum back to line center. Additionally, a broader pulse (narrower optical spectrum) leads to longer relaxation time for this spectral shifting process. The absorption section serves as a compensator for this purpose, because mode coupling within it opposes the spectral narrowing. Indeed, one condition for steady state $^{3}$ in the case of chirp free pulses is

$$
\frac{g}{\Omega_{g}{ }^{2} \tau_{p}{ }^{2}} \simeq \gamma A_{p}{ }^{2},
$$

where $\gamma$ is the effective saturable absorber action and $A_{p}$ is the pulse electric field amplitude. In cases for which the chirp is nonzero other processes such as groupvelocity dispersion and the nonlinear Kerr effect will be involved in determining the relaxation time.

\section{AMPLITUDE FLUCTUATIONS}

Whenever the amplitude fluctuations are small relative to the mean amplitude intensity the power spectrum is influenced mainly by the timing-jitter noise and the results of Section 3 are applicable. The case of strong amplitude fluctuations is studied in this section.

Typically the amplitude fluctuations occur at a rate that is slow relative to the pulse envelope, in which the laser output can be described by a set of discrete coherent pulses. Therefore, assuming that all the pulses are equally shaped but differ in amplitude, we find that $F_{n}(\omega)=\left(A+\delta A_{n}\right) S(\omega)$, where $A$ is the average intensity amplitude, $\delta A_{n}$ is the random variable describing the intensity amplitude fluctuations of the $n$th pulse in the train with zero mean value, and $S(\omega)$ is the Fourier transform of the pulse shape.

We assume that the correlation between timing-jitter and amplitude fluctuations is negligible and that the random amplitude noise possesses an exponential autocorrelation $^{3}$

$$
\left\langle\delta A_{n} \delta A_{m}\right\rangle=\left\langle\delta A^{2}\right\rangle \exp \left(-|n-m| T / \tau_{a}\right),
$$

where $\tau_{a}$ describes the amplitude fluctuation correlation time and $\left\langle\delta A^{2}\right\rangle=\left\langle\delta A_{n}^{2}\right\rangle$. The power spectrum is given by

$$
\begin{aligned}
P_{I}(\omega)=\mid & \left.S(\omega)\right|^{2} \lim _{N \rightarrow \infty} \frac{1}{2 N+1} \sum_{n, m=-N}^{N}\left[A^{2}+\left\langle\delta A^{2}\right\rangle\right. \\
& \left.\times \exp \left(-|n-m| T / \tau_{a}\right)\right] \exp [i \omega T(n-m)] \\
& \times \exp \left[-\frac{\omega^{2}}{2}\left\langle\left(\delta T_{n}-\delta T_{m}\right)^{2}\right\rangle\right]
\end{aligned}
$$

First we study the case of timing-jitter fluctuations modeled by white noise $\left(\tau_{t} \ll T\right)$. Substituting Eq. (17) into Eq. (26), we get

$$
\begin{aligned}
P_{I}(\omega)= & A^{2}|S(\omega)|^{2}\left[\frac{\sinh \left(\omega^{2}\left\langle\Delta T^{2}\right\rangle / 2\right)}{\cosh \left(\omega^{2}\left\langle\Delta T^{2}\right\rangle / 2\right)-\cos (\omega T)}\right. \\
& \left.+\frac{\left\langle\delta A^{2}\right\rangle}{A^{2}} \frac{\sinh \left(\omega^{2}\left\langle\Delta T^{2}\right\rangle / 2+T / \tau_{a}\right)}{\cosh \left(\omega^{2}\left\langle\Delta T^{2}\right\rangle / 2+T / \tau_{a}\right)-\cos (\omega T)}\right] .
\end{aligned}
$$

The influence of the amplitude fluctuations on the power spectrum depends on two parameters: the ratio $\left\langle\delta A^{2}\right\rangle / A^{2}$, which determines the strength of the amplitude noise, and $T / \tau_{a}$, the ratio between the average repetition time and the amplitude fluctuation correlation time. The effect of amplitude fluctuations on the power spectrum [the second term on the right-hand side of Eq. (27)] depends on the ratio between $T / \tau_{a}$ and $\left\langle\Delta T^{2}\right\rangle / T^{2}$. For long amplitude correlation times $\left(T / \tau_{a} \ll\left\langle\Delta T^{2}\right\rangle / T^{2}\right)$, the second term has the same form as the first, and the amplitude fluctuations, even for strong rms fluctuations, have little influence on the power spectrum. For $T / \tau_{a}$ $\gg\left\langle\Delta T^{2}\right\rangle / T^{2}$ and $T / \tau_{a} \gg 1$, the second term is constant but negligible compared with the first term. In the case for which $T / \tau_{a} \gg\left\langle\Delta T^{2}\right\rangle / T^{2}$ but $T / \tau_{a} \ll 1$, the second term can be described by a Lorentzian given by 
$\left(T / 2 \tau_{a}\right) /\left[\left(T / \tau_{a}\right)^{2}+\left(\Delta \omega_{n} T\right)^{2}\right] . \quad$ Amplitude fluctuations mainly influence the tails of the spectra of different harmonics.

Amplitude fluctuations, in the case of white-noise nonstationary timing fluctuation, have significant influence on the power spectrum whenever $T / \tau_{a} \sim\left\langle\Delta T^{2}\right\rangle / T^{2}$. The second term in the square brackets of Eq. (27) is of the same order of magnitude as the first. This term describes a wider Lorentzian than that of the first term, leading to a deviation in the tails of the spectra that decay more slowly.

We shall study the details of the more interesting case involving long timing-jitter correlation times. In this case, for which $\tau_{t} \gg T$, and for high frequencies of the power spectrum, the upper part of Eq. (21) is applicable for the summation over $n$ and $m$ in Eq. (26). The power spectrum has the form of

$$
\begin{aligned}
P_{I}(\omega)= & A^{2}|S(\omega)|^{2}\left(\frac{2 \pi}{\omega^{2}\left\langle\Delta T^{2}\right\rangle}\right)^{1 / 2} \\
& \times \sum_{n=-\infty}^{\infty}\left(\exp \left[-\frac{\left(\Delta \omega_{n} T\right)^{2}}{2 \omega^{2}\left\langle\Delta T^{2}\right\rangle}\right]+\frac{\left\langle\delta A^{2}\right\rangle}{A^{2}}\right. \\
& \times \operatorname{Re}\left\{\exp \left[\frac{\left(T / \tau_{a}+i \Delta \omega_{n} T\right)^{2}}{2 \omega^{2}\left\langle\Delta T^{2}\right\rangle}\right]\right. \\
& \left.\left.\times \operatorname{erfc}\left(\frac{T / \tau_{a}+i \Delta \omega_{n} T}{\sqrt{2 \omega^{2}\left\langle\Delta T^{2}\right\rangle}}\right)\right\}\right)
\end{aligned}
$$

where $\operatorname{Re}(z)$ represents the real part of the complex expression $z$ and $\operatorname{erfc}(z)$ is the complementary error function. ${ }^{5}$ For small amplitude fluctuations the shape of the $n$th harmonic spectra in the power spectrum is given by the $n$th part in the sum of Eq. (28) for small timingjitter rms fluctuations.

As can be expected, for long amplitude correlation times $\left(T / \tau_{a} \ll \sqrt{\left\langle\Delta T^{2}\right\rangle} / T\right)$ the second term of the right hand side of Eq. (28) has a form similar to that of the first term, and amplitude fluctuations are difficult to detect. Amplitude fluctuations are negligible also in the opposite case, i.e., short amplitude correlation times $\left(T / \tau_{a}\right.$ $\left.\gg \sqrt{\left\langle\Delta T^{2}\right\rangle} / T\right)$. In this case the real part of the second term is given by the Lorentzian

$$
\left(\frac{2 \omega^{2}\left\langle\Delta T^{2}\right\rangle}{\pi}\right)^{1 / 2} \frac{T / \tau_{a}}{\left(T / \tau_{a}\right)^{2}+\left(\Delta \omega_{n} T\right)^{2}} .
$$

Its magnitude is negligible compared with that of the Gaussian described in the first term, and it mainly affects the tails of the spectra of these harmonics.

The main influence of strong amplitude fluctuations appears whenever $T / \tau_{a} \sim\left(\left\langle\Delta T^{2}\right\rangle\right)^{1 / 2} / T$. The tails $\left[\Delta \omega_{n} T /\left(2 \omega^{2}\left\langle\Delta T^{2}\right\rangle\right)^{1 / 2} \gg 1\right]$ of the normalized spectra of these harmonics decay as a Lorentzian as in Eq. (29). Even in this case, the main lobe has nearly the same shape and width as without the amplitude fluctuations. Figure 3(a) describes a calculation of the normalized spectra as a function of $\Delta \omega_{n} T /\left(2 \omega^{2}\left\langle\Delta T^{2}\right\rangle\right)^{1 / 2}$ for different values of amplitude fluctuation strength $\left(\left\langle\delta A^{2}\right\rangle / A^{2}\right)$ and cor-
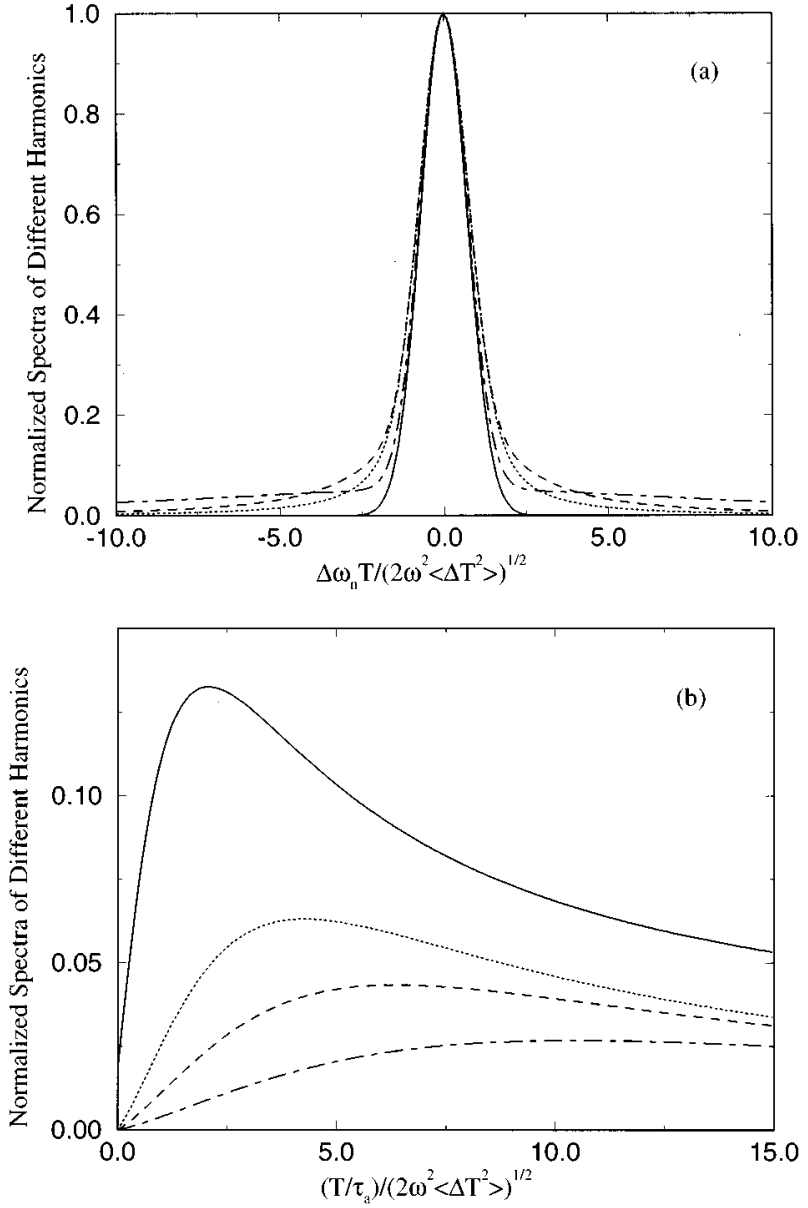

Fig. 3. The effect of amplitude fluctuations on the normalized spectra of long-range timing-jitter correlation time $\tau_{t}$ [the normalized outer parentheses of the summation in Eq. (28)] (a) Versus the scaled frequency offset for different values of scaled amplitude correlation time. The solid curve describes the normalized spectra for $\left\langle\delta A^{2}\right\rangle=0$. The dotted, dashed, and dotted-dashed curves stand for $\left(T / \tau_{a}\right) /\left(2 \omega^{2}\left\langle\Delta T^{2}\right\rangle\right)^{1 / 2}$ equal to 1 , 2 , and 10 , respectively, where maximum amplitude fluctuation was taken into account $\left(\left\langle\delta A^{2}\right\rangle / A^{2}=1\right)$. (b) Versus the scaled amplitude correlation time for several values of scaled frequency offset. The solid curve describes the normalized spectra for $\Delta \omega_{n} T /\left(2 \omega^{2}\left\langle\Delta T^{2}\right\rangle\right)^{1 / 2}=2$. The dotted, dashed, and dotteddashed lines stand for $\Delta \omega_{n} T /\left(2 \omega^{2}\left\langle\Delta T^{2}\right\rangle\right)^{1 / 2}$ equal to 4,6 , and 10, respectively.

relation time $\left(T / \tau_{a}\right) /\left(2 \omega^{2}\left\langle\Delta T^{2}\right\rangle\right)^{1 / 2}$. The solid curve describes the Gaussian shape for negligible rms amplitude fluctuations, $\left\langle\delta A^{2}\right\rangle / A^{2}=0$. This curve also describes long-range amplitude correlation times $\left(T / \tau_{a}\right) /\left(2 \omega^{2}\left\langle\Delta T^{2}\right\rangle\right)^{1 / 2} \ll 1$, or relatively small amplitude correlation times $\left(T / \tau_{a}\right) /\left(2 \omega^{2}\left\langle\Delta T^{2}\right\rangle\right)^{1 / 2} \gg 1$ in the case of strong amplitude fluctuations. The dotted, dashed, and dotted-dashed curves represent the normalized spectra [the normalized outer parentheses of the summation in Eq. (28) are proportional to the normalized spectra] for $\left(T / \tau_{a}\right) /\left(2 \omega^{2}\left\langle\Delta T^{2}\right\rangle\right)^{1 / 2}$ equal to 1,2 , and 10 , respectively, and $\left\langle\delta A^{2}\right\rangle / A^{2}=1$. Even for strong amplitude fluctuations the effect on the power spectrum is relatively weak, since it affects mainly the tails of the spectra of the different harmonics. The main lobe of the spectra are hardly affected, and the scaling of its width as a function of the harmonic number is still expected to hold. 
In Fig. 3(b) a plot of the normalized spectra as a function of the scaled amplitude correlation time $\left(T / \tau_{a}\right) /\left(2 \omega^{2}\left\langle\Delta T^{2}\right\rangle\right)^{1 / 2}$ is shown for different values of the scaled frequency offset in which $\left\langle\delta A^{2}\right\rangle / A^{2}=1$. The solid, dotted, dashed, and dotted-dashed curves represent $\Delta \omega_{n} T /\left(2 \omega^{2}\left\langle\Delta T^{2}\right\rangle\right)^{1 / 2}$ equal to $2,4,6$, and 10 , respectively. For both short and long amplitude correlation times the deviation from the Gaussian is small. For $T / \tau_{a}>1$ the main deviation occurs when $T / \tau_{a} \simeq \Delta \omega_{n} T$ (otherwise the main deviation occurs for $T / \tau_{a} \sim 1$ ).

This theory permits one to find the timing-jitter rms fluctuations from the shape and the width of the spectra for different harmonics of the experimental results and to estimate the correlation time $\tau_{t}$ for nonstationary timing fluctuations. Whenever $n_{\min }=1$, a lower limit for the correlation time can be found. Estimation of the amplitude fluctuations and their correlation time can be achieved in special cases of the amplitude fluctuation strength and its correlation time.

\section{EXPERIMENTAL RESULTS}

Experimental measurements of noise have been made. The laser used is a passively mode-locked quadruple quantum well two-section, GaAs buried heterostructure as described previously. ${ }^{6,7}$ In Fig. 4 the two-section laser with an external cavity is shown. The long laser section is forward biased to provide the gain of the laser. By reverse biasing the short segment of the laser a waveguide saturable (slow) absorber is formed. The central optical frequency is $845 \mathrm{~nm}$ with a repetition rate of $608.5 \mathrm{MHz}$ which is controlled by the external-mirror-to-laser distance.

The power spectrum of the laser intensity was recorded with a fast photodiode and an electronic spectrum analyzer. Its normalized spectra of different harmonics are shown in Fig. 5 as a function of the offset frequency divided by the harmonic number. The solid curve describes the fit to a Gaussian with FWHM equal to 2.83 $\times 10^{-3}$. One can conclude that all the harmonics, starting from the first, can be described by a Gaussian with increasing width proportional to the harmonic number $\left(n_{\min }=1\right)$. Therefore the timing-jitter fluctuations are damped slowly with relaxation time $\tau_{t} / T>(\sqrt{2} / 2 \pi)$ $\times\left(T /\left\langle\Delta T^{2}\right\rangle^{1 / 2}\right) \simeq 1180$, where the rms timing-jitter fluctuation was calculated by fitting its FWHM to that of the Gaussian, yielding $\left\langle\Delta T^{2}\right\rangle^{1 / 2} / T \simeq 1.9 \times 10^{-4}$.

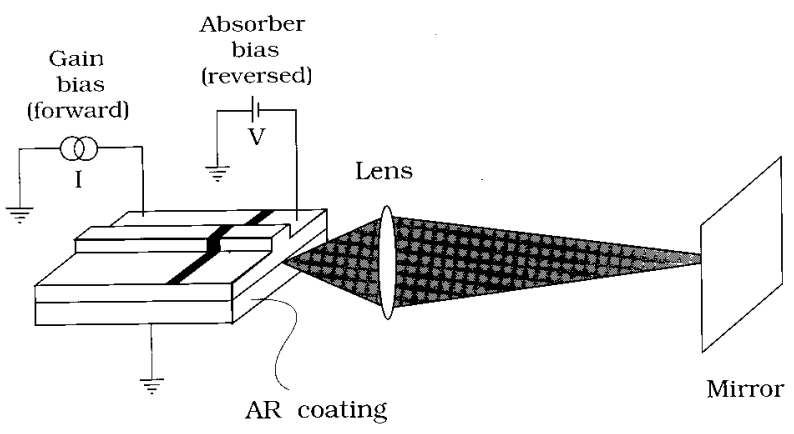

Fig. 4. External cavity configuration for the two-section semiconductor passively mode-locked laser used in the experiment.

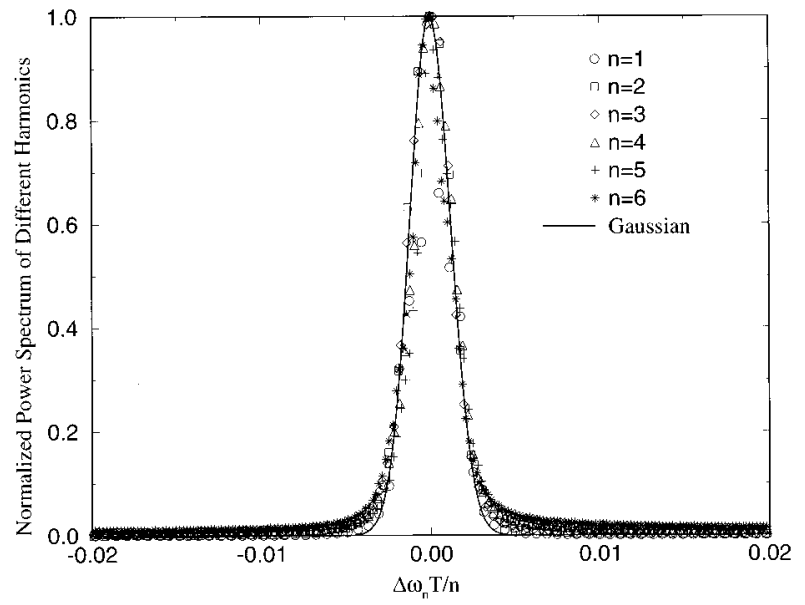

Fig. 5. Normalized intensity spectra of the first six harmonic numbers versus the deviation frequency divided by the harmonic number $n$. The solid curve describes a Gaussian with FWHM of $2.83 \times 10^{-3}$.

Small deviations from the theoretical Gaussian curve appear. Those deviations mainly affect the tails of the normalized spectra and depend on the harmonic number. As the harmonic number increases, the tail's deviation increases. $\left(T / \tau_{a}\right) /\left(2 \omega^{2}\left\langle\Delta T^{2}\right\rangle\right)^{1 / 2}$ decreases as the harmonic number increases, and from Fig. 3(b) and Eq. (28) we conclude that the deviation increases as this parameter decreases only if $\left(T / \tau_{a}\right)<\Delta \omega_{n} T$. Therefore we find that $\tau_{a} / T<8$. It is difficult in this case to obtain more detail of the amplitude rms fluctuations and correlation time from the power spectrum.

\section{CONCLUSION}

We have presented an analysis of the power spectrum of optical pulse trains subject to nonstationary timing-jitter fluctuations. This theory is suitable for passively modelocked lasers in which external modulation is absent. Only the statistical properties of timing-jitter fluctuations between successive neighboring pulses were assumed to be stationary, i.e., independent of the origin of time. It is shown that the discrete lines and the $\omega^{2}$ increase of the side-band peak values (characteristic of the low-frequency power spectrum for stationary timing-jitter fluctuations, as in actively mode-locked lasers) are not valid in this case.

When timing-jitter fluctuations between successive neighboring pulses are uncorrelated in time, Lorentzianshaped spectra at different harmonics with FWHM proportional to the square of the harmonic number will result. Effects of correlations between the timing fluctuations of different pulses in the train were studied as well. These correlations between timing fluctuations tend to produce spectra at different harmonics, which are both Gaussian in shape and have FWHM's that increase linearly with harmonic number (for harmonic number greater than $\left.n_{\min }\right)$. As the ratio of the correlation time to repetition time $\left(\tau_{t} / T\right)$ increases compared with the ratio of the repetition time to $\mathrm{rms}$ timing-jitter between neighboring pulses $\left(T /\left\langle\Delta T^{2}\right\rangle^{1 / 2}\right)$, more harmonics will have the Gaussian shape and linear dependence of the FWHM on 
frequency. The connection between the correlation time and the relaxation time that results from the coupling between the modes was discussed briefly.

Amplitude fluctuations were found to have only a small effect on the power spectrum. Small amplitude fluctuations or strong fluctuations with long or short amplitude correlation times both have little effect on the shape of the spectra. Other cases of strong amplitude fluctuations mainly extend the wings of the spectra around different harmonic numbers.

Experimental results for an external cavity passively mode-locked semiconductor laser were presented. The first six measured harmonics had Gaussian shapes with the FWHM linearly dependent on harmonic number. This indicates a long relaxation time for the energy transfer between the modes or the relaxation time of the relative phases among the modes.

Finally we note that this theory can be applied to active mode-locking systems whenever the external modulation source is unstable or possesses the dominant timing fluctuation that results from the sum of many processes, i.e., nonstationary timing-jitter fluctuations.

\section{ACKNOWLEDGMENTS}

This research was supported by the National Science Foundation and the Advanced Research Projects Agency.

\section{REFERENCES}

1. D. von der Linde, "Characterization of the noise in continuously operating mode-locked lasers,” Appl. Phys. B 39, 201217 (1986).

2. D. Eliyahu, R. A. Salvatore, and A. Yariv, "Noise characterization of pulse train generated by actively mode-locked lasers," J. Opt. Soc. Am. B 13, 1619-1626 (1996).

3. H. A. Haus and A. Mecozzi, "Noise of mode-locked lasers," IEEE J. Quantum Electron. 29, 983-996 (1993).

4. A. Yariv, Optical Electronics, 4th ed. (Saunders, Philadelphia, Pa., 1991).

5. I. S. Gradshteyn and I. M. Ryzhik, Table of Integrals, Series and Products, 4th ed. (Academic, New York, 1965).

6. R. A. Salvatore, T. Scharns, and A. Yariv, "Pulse characteristics of passively mode-locked diode lasers," Opt. Lett. 20, 737-739 (1995).

7. S. Sanders, A. Yariv, J. Paslaski, J. E. Ungar, and H. A. Zarem, "Passive mode locking of a two-section multiple quantum well laser at harmonics of the cavity round-trip frequency," Appl. Phys. Lett. 58, 681-683 (1991). 